\title{
Tailoring insoluble nanobelts into soluble anti-UV nanopotpourris
}

\author{
Jinmin Wang, ${ }^{a}$ Xiao Wei Sun, ${ }^{* a}$ Zhihui Jiao, ${ }^{a}$ Eugene Khoo, ${ }^{b}$ Pooi See Lee, ${ }^{* b}$ Jan Ma \\ and Hilmi Volkan Demir ${ }^{a}$
}

Received 31st July 2011, Accepted 5th September 2011
DOI: 10.1039/c1nr10979k

Soluble, transparent and anti-UV nanopotpourris have been prepared by tailoring long nanobelts. The strains and layered structures facilitate the breaking of the as-synthesized nanobelts under an applied mechanical action. The developed tailoring process of nanobelts is a general top-down secondary processing of layered nanostructures at the nanoscale level, which can be expended to the modifications of layered nanowires, nanotubes and hierarchical nanostructures. By tailoring, the size, morphology and solubility are modified, which may open up an area of advanced processing of nanomaterials and hint at some potential applications. Because of the excellent solubility of the tailored nanopotpourris, they are easily dispersed in cosmetics or polymer films, which are quite useful for some anti-UV protection applications, such as anti-UV sunscreen creams and anti-UV window films for vehicles and buildings.

\section{Introduction}

Synthesis and assembly of nanostructures have attracted significant interest due to the size and dimension dependent optical, electronic, and magnetic properties. ${ }^{1}$ The assembly of nanoscale building blocks into larger complex structures is one of the most promising yet challenging fields of nanoscience and nanotechnology. ${ }^{2}$ The assembled structures could facilitate a deeper understanding of the "bottom-up" processes, a potential method of discovering new special properties, and a powerful way of fabricating complex nanodevices, such as solar cells, nextgeneration microelectronic and optoelectronic devices. ${ }^{3}$ Compared with the bottom-up assembly of nanostructures, "topdown" approaches are usually employed to fabricate nanostructures from bulk materials by some physical treatments, such as crystallization of amorphous solids, ${ }^{4}$ e-beam lithography, ${ }^{5}$ and ball milling. ${ }^{6}$ However, a more precise top-down process at the nanoscale level, that is a secondary processing of nanostructures, will be more difficult, and hence it has not attracted enough attention yet. Only a few studies have reported that carbon nanotubes could be opened by oxidation using oxygen ${ }^{7}$ or concentrated acid. ${ }^{8}$ The direct top-down "tailoring" of nanostructures instead of bulk materials should be developed intensively to prepare new nanoscale units and explore their properties.

${ }^{a}$ School of Electrical and Electronic Engineering, Nanyang Technological University, Singapore, 639798, Singapore. E-mail: exwsun@ntu.edu.sg; Tel: $+65-67905369$

${ }^{b}$ School of Materials Science and Engineering, Nanyang Technological University, Singapore, 639798, Singapore. E-mail: pslee@ntu.edu.sg; Tel: $+65-67906661$
It is well-known that the quantum confinement effect of nanostructures results in unique electrical, optical, magnetic and thermoelectric properties. ${ }^{9}$ To achieve these special properties, nanostructures with quantum sizes must be synthesized first. However, it may be quite difficult to synthesize quantum-sized nanostructures for some materials. Hence, a top-down secondary processing of nanostructures becomes useful and significant to create quantum-sized nanostructures and to explore their special properties. By tailoring nanostructures, not only the size, but also the morphology, surface, crystallinity, band-gap and defect could be modified, which provides the possibility to generate special properties from the tailored nanoscale units.

Herein, we demonstrate a method of tailoring insoluble nanobelts into completely soluble nanopotpourris with an antiUV property.

\section{Experimental procedures}

$\mathrm{NaV}_{3} \mathrm{O}_{8} \cdot 0.9 \mathrm{H}_{2} \mathrm{O}$ nanobelts were synthesized by a hydrothermal process similar to our previously reported procedure. ${ }^{10}$ In a typical synthesis, $0.234 \mathrm{~g}(2 \mathrm{mmol})$ of ammonium metavanadate $\left(\mathrm{NH}_{4} \mathrm{VO}_{3}\right)$ and $0.468 \mathrm{~g}(8 \mathrm{mmol})$ of $\mathrm{NaCl}$ were added into $20 \mathrm{~mL}$ of deionized water with stirring for $15 \mathrm{~min}$ to form a clear yellow solution. Hydrochloric acid $(\mathrm{HCl})$ was dropped into the solution until a $\mathrm{pH}$ of 3.2 , an orange solution was formed. The orange solution was transferred into a Teflon-lined autoclave for a hydrothermal treatment in an oven at $180^{\circ} \mathrm{C}$ for $24 \mathrm{~h}$. After cooling down to room temperature and washing with deionized water, the brownish sponge-like nanobelts were obtained. The tailoring of the as-synthesized nanobelts into nanopotpourris was realized by stirring in deionized water for 3 days. At the beginning, the nanobelts were completely insoluble 
and subsequently formed a brown suspension; after the stirring, the suspension became clearer and clearer and the colour changed to yellow; at last, the nanobelts were completely dissolved and a transparent yellow sol was obtained.

The as-prepared nanobelts and tailored nanopotpourris were characterized by X-ray powder diffraction (XRD, Shimadzu), using $\mathrm{Cu} \mathrm{K} \alpha(\lambda=0.15406 \mathrm{~nm})$ radiation, field-emission scanning electron microscopy (FESEM, JSM-6700F), transmission electron microscopy (TEM, JEM-2010) with energy-dispersive X-ray spectroscopy (EDS). Thermogravimetric analysis (TGA) was carried out using a TA Instruments TGA Q500 with a heating rate of $10{ }^{\circ} \mathrm{C} \mathrm{min}{ }^{-1}$. The $\mathrm{UV}-\mathrm{V}$ is absorption and transmittance spectra were measured using a UV-Vis spectrophotometer (JESCO V670).

\section{Results and discussion}

The XRD patterns of the as-synthesized nanobelts are shown in Fig. 1a. For the as-synthesized nanobelts, one strong peak at $\sim 10.56^{\circ}$ and one weak peak at $\sim 21.12^{\circ}$ (around two times of $10.56^{\circ}$ ) are detected, corresponding to the preferential orientation along $(00 l)$ crystal planes. This is similar to the XRD pattern of vanadium oxide hydrate. ${ }^{11}$ It is difficult to identify the nanobelts according to the two peaks. So the nanobelts were annealed at $400{ }^{\circ} \mathrm{C}$ in air to check the possible change. It can be found that some new peaks are detected, thus it becomes possible to identify the annealed nanobelts. However, there are a few compounds which have similar XRD patterns to those of the annealed nanobelts. Considering the used chemicals and possible products in the hydrothermal reaction, only $\mathrm{NaV}_{3} \mathrm{O}_{8}$ (JCPDS 49-0997), $\mathrm{Na}_{0.33} \mathrm{~V}_{2} \mathrm{O}_{5}$ (JCPDS 24-1155 or 86-0120), $\beta-\mathrm{Na}_{0.33} \mathrm{~V}_{2} \mathrm{O}_{5}$ (JCPDS
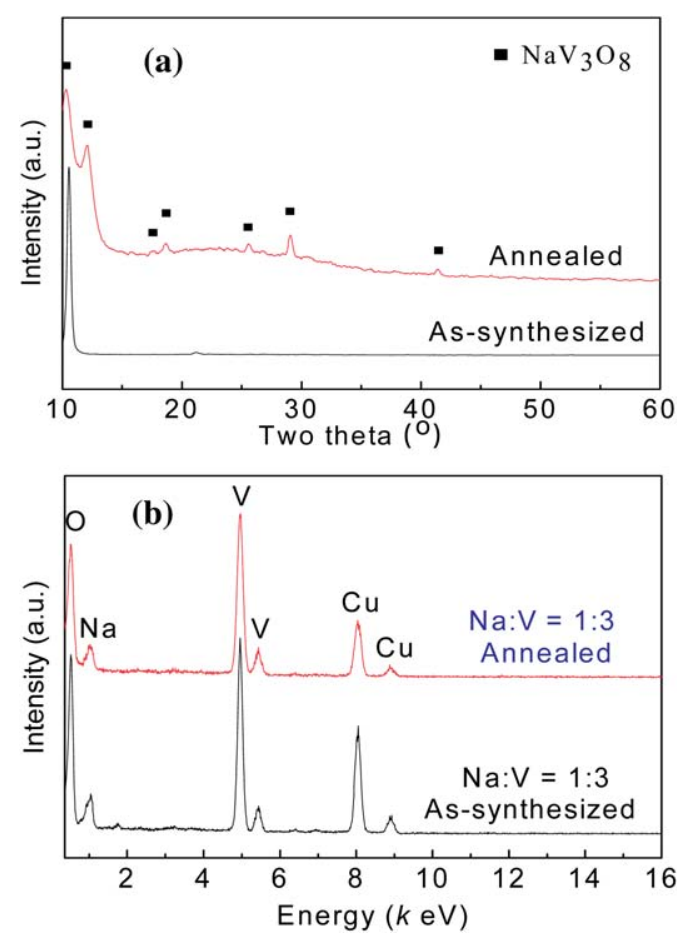

Fig. 1 (a) XRD patterns of the as-synthesized nanobelts and the annealed nanobelts at $400{ }^{\circ} \mathrm{C}$ in air and (b) EDS spectra of the assynthesized nanobelts and the annealed nanobelts at $400{ }^{\circ} \mathrm{C}$ in air.
49-0998) and $\mathrm{Na}_{0.76} \mathrm{~V}_{6} \mathrm{O}_{15}$ (JCPDS 75-1653) are possible annealed products. All these compounds contain $\mathrm{Na}$ and $\mathrm{V}$ elements with different atomic ratios. Hence, EDS was employed to check the atomic ratio, the corresponding EDS spectra are shown in Fig. 1b. The atomic ratio of $\mathrm{Na}: \mathrm{V}$ is found to be $\sim 1: 3$ for both of the as-synthesized and annealed nanobelts according to the EDS analysis. Thus, the only possible composition among the candidates for annealed nanobelts is $\mathrm{NaV}_{3} \mathrm{O}_{8}$. Considering the used chemicals in the hydrothermal reaction and the possible products again, it is believed that the as-synthesized nanobelts contain hydrate in which $\mathrm{H}$ cannot be detected in the EDS result.

To determine the amount of hydrate in the as-synthesized nanobelts, TGA was used to calculate the ratio of hydrate. The TGA curve is shown in Fig. 2 . The first weight loss before $\sim 131$ ${ }^{\circ} \mathrm{C}$ is caused by the removal of physically absorbed surface water. However, the second weight loss between 212 to $\sim 337^{\circ} \mathrm{C}$ is due to the removal of chemically bound hydrate within the nanobelts. Hence, the second weight loss can be used to calculate the ratio of hydrate within the nanobelts. The calculated weight loss of $4.9 \%$ corresponds to $\mathrm{NaV}_{3} \mathrm{O}_{8} \cdot 0.9 \mathrm{H}_{2} \mathrm{O}$ which has been reported by Spahr et al. ${ }^{12}$ Similar hydrated sodium vanadates $\left(\mathrm{NaV}_{3} \mathrm{O}_{8} \cdot 0.7 \mathrm{H}_{2} \mathrm{O}, \mathrm{NaV}_{3} \mathrm{O}_{8} \cdot 1.5 \mathrm{H}_{2} \mathrm{O}\right)^{13-15}$ have also been reported. So the as-synthesized nanobelts (without annealing) is $\mathrm{NaV}_{3} \mathrm{O}_{8} \cdot 0.9 \mathrm{H}_{2} \mathrm{O}$.

Fig. 3 shows the morphologies of the as-synthesized nanobelts. The sponge-like nanobelts are ultra-long, with lengths of up to a few millimetres (Fig. 3a). Their typical widths and thicknesses are $\sim 50$ to 500 and $\sim 10$ to $100 \mathrm{~nm}$, respectively. The lengthwidth ratio (corresponding the aspect ratio of nanowires) of the nanobelts is over 1000. The TEM images (Fig. 3d and e) also clearly show the belt-like structure and the sides of nanobelts.

The morphologies of the tailored nanopotpourris are shown in Fig. 4. Various morphologies, including nanosheets (NSs), nanorods (NRs), nanoparticles (NPs) and quantum dots (QDs) are observed. These nanostructures have different particle sizes: nanosheets with widths of hundreds of nanometres and thicknesses of tens of nanometres, nanorods with lengths of hundreds of nanometres and diameters of tens of nanometres, nanoparticles with diameters of tens of nanometres and QDs with diameters of $\sim 2$ to $6 \mathrm{~nm}$. A wide particle size distribution is obtained after the tailoring.

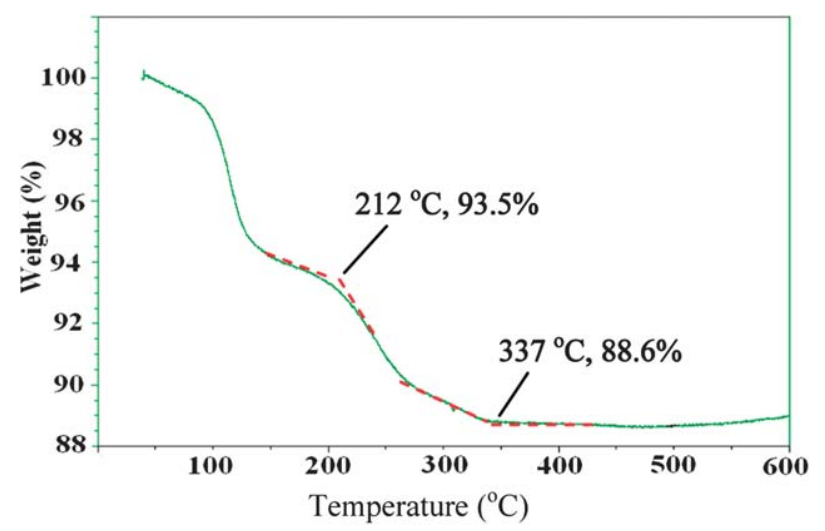

Fig. 2 TGA curve of the as-synthesized nanobelts measured in air with a heating rate of $10{ }^{\circ} \mathrm{C} \mathrm{min}^{-1}$. 


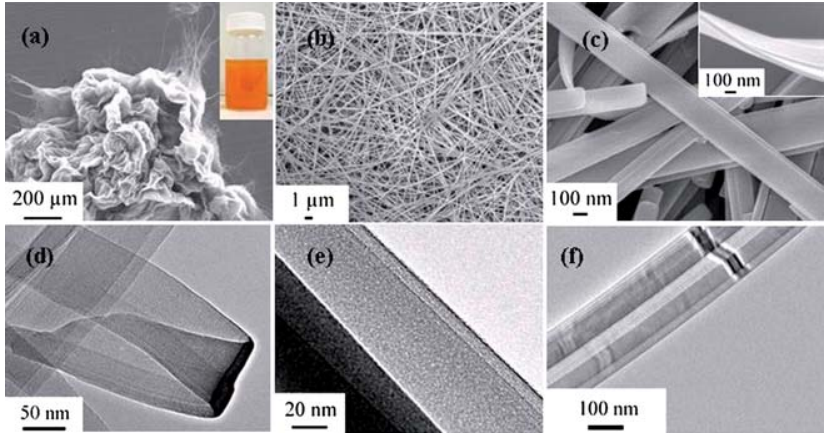

Fig. 3 (a-c) FESEM and (d-f) TEM images of the as-synthesized nanobelts. Inset of (a): a photo of the brown suspension containing nanobelts; inset of (c): a FESEM image clearly showing the belt-like and layered structures.
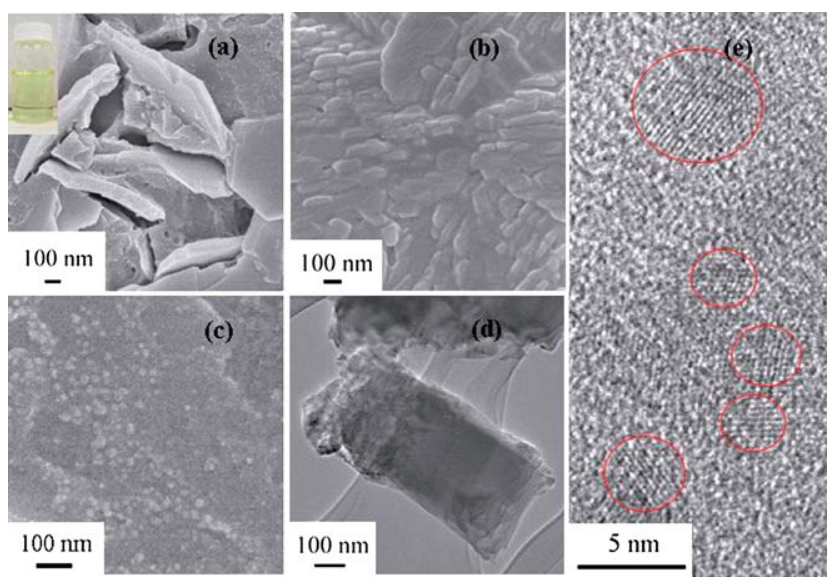

Fig. 4 (a-c) FESEM, (d) TEM and (e) HRTEM images of the tailored soluble nanopotpourris. Inset of (a): a photo of the yellow sol containing nanopotpourris. Quantum dots can be observed in (e) (see the areas surrounded by the red circles).

It is surprising that the as-synthesized nanobelts can be cut into completely soluble nanopotpourris by stirring in water, which forms a transparent sol. By conventional top-down processes (for example, ball-milling), some other nanobelts, such as $\mathrm{In}_{2} \mathrm{O}_{3}$ nanobelts that are highly flexible, ${ }^{16}$ can only be partially dissociated into nanostructures from a bulk material and considerable residue is still bulk, which usually forms a precipitate or suspension in water. The reason for complete breaking of nanobelts into nanopotpourris in our case can be found by analyzing the special structure of the as-synthesized nanobelts. The nanobelts have a quite large length-width (thickness) ratio. Thus a long structure easily causes bending due to surface charge, space charge or surface stress, ${ }^{17}$ resulting in considerable strains, ${ }^{18}$ which are common in nanobelts and nanowires. The strains were also found in the as-synthesized nanobelts (shown in Fig. 5a and $\mathrm{b}$ ). The strains facilitate the breaking of nanobelts; however the strain is not the only factor for the formation of nanopotpourris. The as-synthesized $\mathrm{NaV}_{3} \mathrm{O}_{8} \cdot 0.9 \mathrm{H}_{2} \mathrm{O}$ nanobelts are layered structures (shown in Fig. $3 \mathrm{c}$ and $\mathrm{f}$ and $5 \mathrm{c}$ ), similar layered structures of sodium vanadium oxide hydrate have been reported by Spahr and Novák et al., ${ }^{12-14}$ Durupthy and Steunou et al. ${ }^{15}$ The layered structures further facilitate the breaking (for
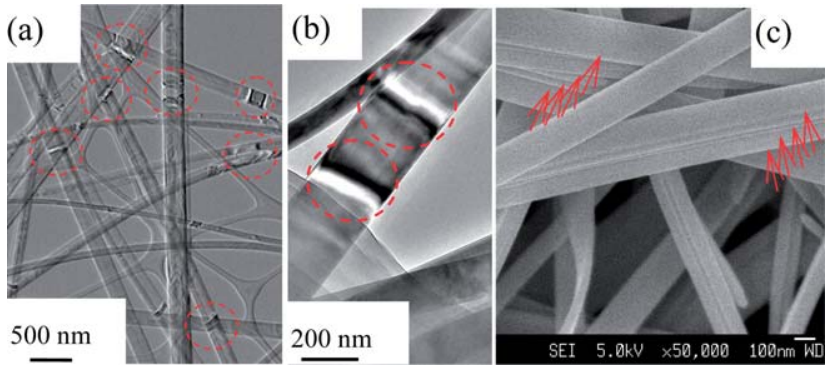

Fig. 5 ( $a$ and b) TEM and (c) FESEM images of the as-synthesized nanobelts. The ripple-like contrast (see the areas surrounded by the red circles) observed in the TEM images ( $a$ and $b$ ) is due to strains resulting from the bending of the nanobelts; the layered structures can be seen at the arrowheads.

example, interlaminar exfoliation) under mechanical treatments because the binding force in the interfaces between adjacent layers is weaker than elsewhere.

The schematic illustration for the tailoring process of nanobelts is shown in Fig. 6. When the fragile nanobelts were vigorously stirred in water, they were cut into shorter and shorter nanobelts first, and then became NSs (observed in Fig. 4a) by further stirring. Besides this kind of tailoring, the NSs can be further cut along the edges, resulting in the formation of NRs (observed in Fig. 4b). And the NRs can be further cut into NPs and QDs (observed in Fig. 4c and e). Certainly, some NRs, NPs and QDs can also be formed accompanying the formation of nanosheets during the breaking of nanobelts. Furthermore, the smallest QDs can be further generated by breaking the nanosheets, nanorods and nanoparticles. In a word, smaller nanoscale units are generated by breaking larger nanostructures under the mechanical action, resulting in completely soluble nanopotpourris with various morphologies.

The tailored soluble nanopotpourris show wide and strong UV absorption and weak purple-blue visible absorption (Fig. 7a). From $\sim 500 \mathrm{~nm}$ to the UV absorption section, there is a consecutive increase in the UV-Vis absorption, showing a strong and broad-spectrum anti-UV property; also implying the wide size distribution of the tailored nanopotpourris, which is consistent with the FESEM and TEM observations. Fig. 7b shows the transmittance spectrum of the nanopotpourris. It can be seen that there is almost a complete transmittance from 500 to $800 \mathrm{~nm}$, corresponding to the extra-low absorption in the

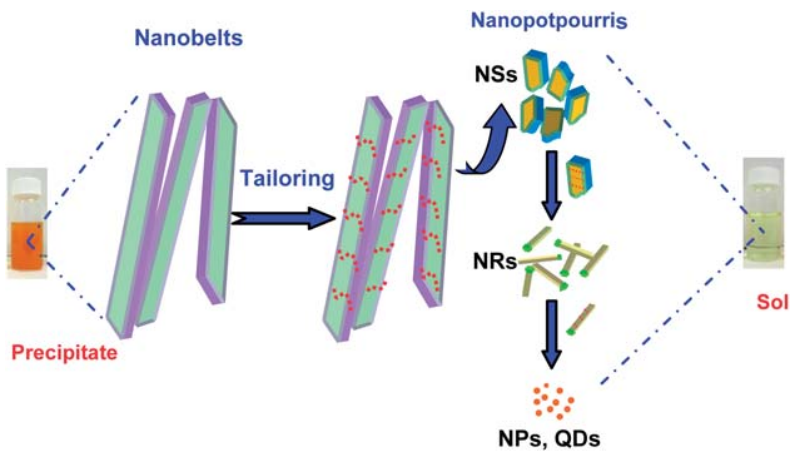

Fig. 6 Schematic illustration for the tailoring process from nanobelts to nanopotpourris. 

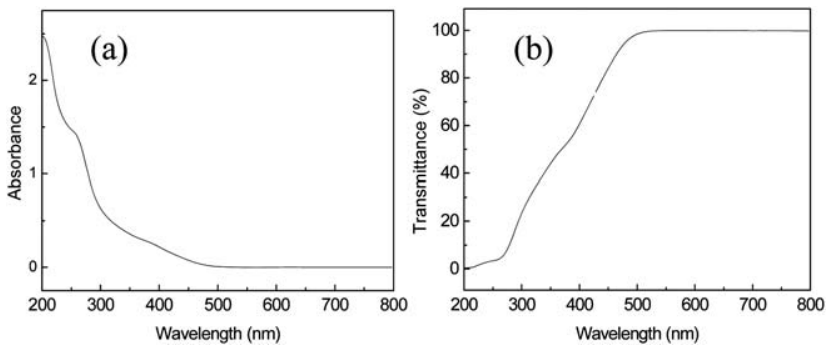

Fig. 7 UV-Vis absorption of the tailored soluble nanopotpourris.

absorption curve. Both the high transparency and strong antiUV properties make the tailored soluble nanopotpourris an important application in anti-UV materials, such as sunscreen creams and anti-UV window films for vehicles and buildings.

\section{Conclusion}

A secondary-processing of nanostructures has been developed to successfully tailor insoluble $\mathrm{NaV}_{3} \mathrm{O}_{8} \cdot 0.9 \mathrm{H}_{2} \mathrm{O}$ nanobelts into complete soluble nanopotpourris with a strong and broadspectrum anti-UV property. The strains and layered structures facilitate the breaking of the as-synthesized nanobelts under the applied mechanical action. The developed tailoring process of nanobelts is a general top-down secondary-processing of layered nanostructures at the nanoscale level, which can be extended to layered nanowires, nanotubes and hierarchical nanostructures modification. By tailoring, the size, morphology and solubility are modified, which may open up an area of advanced processing of nanomaterials and hint at some potential applications. For example, the tailored nanopotpourris may be dispersed in cosmetics or polymer films due to their good solubility, which is quite useful for some anti-UV protection applications, such as anti-UV sunscreen creams and anti-UV window films for vehicles and buildings.

\section{Notes and references}

1 (a) X. G. Peng, L. Manna, W. D. Yang, J. Wickham, E. Scher, A. Kadavanich and A. P. Alivisatos, Nature, 2000, 404, 59; (b) X. F. Duan, Y. Huang, Y. Cui, J. Wang and C. M. Lieber, Nature, 2001, 409, 66; (c) G. M. Whitesides and B. Grzybowski, Science, 2002, 295, 2418; (d) Y. Sun and Y. Xia, Science, 2002, 298, 2176; (e) X. Wang, J. Zhuang, Q. Peng and Y. D. Li, Nature, 2005, 437, 121; (f) M. J. Bierman, Y. K. A. Lau, A. V. Kvit, A. L. Schmitt and
S. Jin, Science, 2008, 320, 1060; $(g)$ H. G. Yang, C. H. Sun, S. Z. Qiao, J. Zou, G. Liu, S. C. Smith, H. M. Cheng and G. Q. Lu, Nature, 2008, 453, 638; $(h)$ H. G. Yang, G. Liu, S. Z. Qiao, C. H. Sun, Y. G. Jin, S. C. Smith, J. Zou, H. M. Cheng and G. Q. Lu, J. Am. Chem. Soc., 2009, 131, 4078; (i) S. A. Morin, M. J. Bierman, J. Tong and S. Jin, Science, 2010, 328, 476; (j) Y. Fang, D. Gu, Y. Zou, Z. X. Wu, F. Y. Li, R. C. Che, Y. H. Deng, B. Tu and D. Y. Zhao, Angew. Chem., Int. Ed., 2010, 49, 7987; $(k)$ J. M. Wang, E. Khoo, J. Ma and P. S. Lee, Chem. Commun., 2010, 46, 2468; (l) Y. Qin, A. Pan, L. F. Liu, O. Moutanabbir, R. B. Yang and M. Knez, ACS Nano, 2011, 5, 788; (m) C.-A. Lin, D.-S. Tsai, C.-Y. Chen and J.-H. He, Nanoscale, 2011, 3, 1195.

2 P. S. Weiss, ACS Nano, 2008, 2, 1085.

3 (a) Y. Huang, X. F. Duan, Q. Q. Wei and C. M. Lieber, Science, 2001, 291, 630; (b) Y. Cui, Q. Wei, H. Park and C. M. Lieber, Science, 2001, 293, 1289; (c) F. Gao, Q. Y. Lu, S. H. Xie and D. Y. Zhao, $A d v$. Mater., 2002, 14, 1537; (d) L.-S. Li and A. P. Alivisatos, $A d v$. Mater., 2003, 15, 408; (e) G. E. Cheng, J. M. Wang, X. W. Liu and K. X. Huang, J. Phys. Chem. B, 2006, 110, 16208; $(f)$ M. L. Shi, W. Su and H. Matsui, Nanoscale, 2010, 2, 2373.

4 (a) K. Lu, J. T. Wang and W. D. Wei, J. Appl. Phys., 1991, 69, 522; (b) K. Lu, Mater. Sci. Eng., R, 1996, 16, 161.

5 K. Mølhave, D. N. Madsen, A. M. Rasmussen, A. Carlsson, C. C. Appel, M. Brorson, C. J. H. Jacobsen and P. Bøggild, Nano Lett., 2003, 3, 1499.

6 F. Q. Guo and K. Lu, Metall. Mater. Trans. A, 1997, 28, 1123.

7 P. M. Ajayan, T. W. Ebbesen, T. Ichihashi, S. Lijima, K. Tanigaki and H. Hiura, Nature, 1993, 361, 333.

8 S. C. Tsang, Y. K. Chen, P. J. F. Harris and M. L. H. Green, Nature, 1994, 372, 159.

9 Y. Xia, P. D. Yang, Y. Sun, Y. Wu, B. Mayers, B. Gates, Y. Yin, F. Kim and H. Yan, Adv. Mater., 2003, 15, 353.

10 E. Khoo, J. M. Wang, P. S. Lee and J. Ma, J. Mater. Chem., 2010, 20, 8368.

11 Y. Yang, Q. Y. Zhu, A. P. Jin and W. Chen, Solid State Ionics, 2008, 179, 1250.

12 M. E. Spahr, P. Novák, W. Scheifele, O. Haas and R. Nesper, J. Electrochem. Soc., 1998, 145, 421.

13 P. Novák, V. Shklovei and R. Nesper, Z. Phys. Chem., 1994, 185, 51.

14 P. Novák, W. Scheifele and O. Haas, J. Power Sources, 1995, 54, 479.

15 O. Durupthy, N. Steunou, T. Coradin, J. Maquet, C. Bonhomme and J. Livage, J. Mater. Chem., 2005, 15, 1090.

16 Z. L. Wang, R. P. Gao, Z. W. Pan and Z. R. Dai, Adv. Eng. Mater., 2001, 3, 657.

17 (a) X. Y. Kong and Z. L. Wang, Nano Lett., 2003, 3, 1625; (b) C. Majidi, Z. Chen, D. J. Srolovitz and M. Haataja, J. Mech. Phys. Solids, 2010, 58, 73; (c) J. K. Jian, Z. H. Zhang, Y. P. Sun, M. Lei, X. L. Chen, T. M. Wang and C. Wang, J. Cryst. Growth, 2007, 303, 427.

18 (a) Z. W. Pan, Z. R. Dai and Z. L. Wang, Science, 2001, 291, 1947; (b) Z. L. Wang and J. H. Song, Science, 2006, 312, 242; (c) X. Wu, P. Jiang, Y. Ding, W. Cai, S.-S. Xie and Z. L. Wang, Adv. Mater., 2007, 19, 2319; (d) S. P. Fu, C. J. Yu, T. T. Chen, G. M. Hsu, M. J. Chen, L. C. Chen, K. H. Chen and Y. F. Chen, Adv. Mater., 2007, 19, 4524. 